\title{
Corrosion dans les centrales nucléaires à eau pressurisée $\left(^{*}\right)$
}

\author{
Pierre Saint-Paul
}

EDF/GDL, 21 Allée privée, 93206 Saint-Denis Cedex, France

\begin{abstract}
Résumé. - Dans une centrale à eau pressurisée, les matériaux soumis aux plus hautes températures sont bien évidemment les matériaux de gainage du combustible qui sont en zircaloy. Compte tenu des inconvénients qui découleraient de nombreuses ruptures de gaine par corrosion, les conditions d'utilisation sont telles que les marges prises vis-à-vis de la corrosion généralisée ou sous contrainte sont très largement suffisantes pour qu'aucune rupture due à ce type de dommage ne se soit encore produite en fonctionnement. Cependant, au niveau des internes du cœur, des fissurations d'aciers inoxydables austénitiques non sensibilisés à la corrosion intergranulaire, soumis au flux thermique et à des contraintes de tension se sont produites récemment dans le milieu réducteur. Le mécanisme de fissuration de ces matériaux, non encore élucidé, est probablement à relier à la corrosion fissurante assistée par l'irradiation. La fissuration des tubes de générateur de vapeur en alliage de nickel dans le milieu primaire est connue depuis longtemps et contraint les exploitants à de très nombreux contrôles, à des bouchages de tube et même au remplacement des générateurs de vapeur. Ce qui est nouveau, c'est que d'autres pièces, plus massives, du circuit primaire, constituées du même alliage et soumises à des contraintes importantes de tension, commencent également à se fissurer. Ces quelques cas de fissuration conduisent à augmenter notablement les contrôles et les opérations de maintenance pour toutes les pièces en alliage de nickel. Dans le milieu secondaire, il semble que la présence de plomb qui peut se concentrer sur les tubes de générateur de vapeur accélère notablement la fissuration de l'alliage 600 en milieu pollué et conduise dans certains cas à une fissuration mixte (inter et transgranulaire). Ce rôle accélérateur du plomb dans la corrosion sous contrainte des alliages austénitiques en milieu alcalin ou acide a été confirmé en laboratoire.
\end{abstract}

\begin{abstract}
In a PWR plant, the materials in contact with the higher temperature are, of course, the materials of fuel cladding : zircaloy. Because the drawbacks which should follow the cladding breakage by corrosion, we have choosen such conditions that the generalized corrosion or the stress corrosion cracking are absolutely avoided. Today, any breakage, due to this damage have been noted on the PWR plants. However, in-core components, intergranular stress corrosion cracking of non-sensitized stainless steels under irradiation exposure has been reported recently in french PWR plants. The cracking mechanism is not yet elucidated, but it is probably relative to irradiation assisted stress corrosion cracking. The cracking of steam generator tubing alloy 600 in the primary side, since very long time, is known to lead to very numerous NDT, tube plugging and even to the steam generator replacements. But, a new phenomenon is observed; the stress corrosion cracking of other equipments in alloy 600 used in the primary side like pressurizer or vessel head penetration. This few examples of cracking lead to increase appreciably the NDT and the maintenance. In the secondary side, it seems that the lead can concentrate on the tubing and allows, sometimes, to obtain mixte (inter and intragranular) mode cracking. On the other hand it seems that in presence of lead the corrosion of alloy 600 is very accelerated. This possibility to accelerate the stress corrosion cracking in alkalizing or acidic medium has been reproduced in laboratories.
\end{abstract}

(*) Keynote lecture. 


\section{Introduction.}

Les conditions sévères de fonctionnement liées à la température et à la pression ainsi que les risques importants de pollution des fluides véhiculés dans les centrales thermiques productrices d'électricité, justifient que les problèmes de corrosion des matériaux soient un souci primordial pour l'exploitant.

Lorsqu'il s'agit de nucléaire et que celui-ci participe à hauteur de $75 \%$ de la production, on comprend que les dégradations du matériel par corrosion qui entrainent des indisponibilités coûteuses ou qui risquent de détériorer la sûreté, doivent être limitées le plus possible. Or, et ceci se vérifie sur les centrales qui atteignent ou dépassent les 10 années de fonctionnement, alors qu'il est envisagé de les faire fonctionner 40 ans, la corrosion est probablement un des facteurs les plus notables de ce que l'on peut appeler le vieillissement des centrales.

La nécessité de connaître le comportement à long terme des matériels, a imposé la mise en œuvre d'un programme considérable de contrôles non destructifs lors des visites annuelles, ainsi que le lancement d'études de laboratoire pour comprendre les dégradations observées sur les pièces retirées du service pour expertise.

Ces contrôles nombreux et de plus en plus fins, ont permis de mettre en exergue des problèmes peu connus ou dont la probabilité d'apparition était très faible.

Il est certain que dès le démarrage des premières tranches, aux Etats-Unis en particulier, des cas de corrosion de matériel ont été rencontrés et souvent présentés dans les Congrès. Aussi, cet exposé ne cherche pas à être exhaustif et à présenter les 15 années de retour d'expérience, mais s'attache à montrer quelques problèmes de corrosion ou de dégradation parmi les plus récents qui ont nécessité, pour la plupart, la mise en route de nouveaux programmes de recherche afin de comprendre et de résoudre les difficultés rencontrées.

\section{Le circuit primaire.}

2.1 LE COMBUSTIBLE. - Dans une centrale à eau pressurisée, les matériaux soumis aux plus hautes températures sont bien évidemment les matériaux de gainage du combustible. Du fait de ses propriétés spécifiques : faible absorption des neutrons, bonnes propriétés mécaniques et tenue à la corrosion, le zircaloy 4 est largement utilisé dans les assemblages combustibles.

Les risques de corrosion de ce matériau sont essentiellement de deux types. Le premier est un risque de corrosion sous contrainte ; sachant que le combustible se fracture, change de densité, produit des gaz de fission dont des iodes et qu'il peut y avoir des variations brutales de température, une intéraction alors possible entre le combustible et la gaine peut produire des contraintes de tension insuffisamment accommodées par le fluage du zircaloy 4. Dans ces conditions, un phénomène de fissuration (contraintes de tension en présence d'iode) peut apparaître. Le second type, compte tenu de la grande affinité du zirconium pour l'oxygène, est la corrosion généralisée des surfaces en contact avec le milieu primaire [1, 2].

Dans les réacteurs de puissance français, le combustible nucléaire est actuellement utilisé pendant 3 ou 4 campagnes d'irradiation ce qui conduit à des épuisements spécifiques pouvant atteindre $42 \mathrm{GWj} / \mathrm{tU}$. Dans ces conditions, les couches d'oxyde sont généralement adhérentes et d'épaisseur de l'ordre de $30 \mu \mathrm{m}$ ( 3 cycles) ou $45 \mu \mathrm{m}$ (4 cycles) au point chaud des crayons de combustible. Loxydation des composants de la structure des assemblages est un peu plus faible : environ 15 à $20 \mu \mathrm{m}$ pour les tubes guides ( 3 cycles) et les grilles les plus oxydées.

L'oxydation entraine une hydruration concomitante de l'alliage sous-jacent; on admet généralement que la teneur en hydrogène est de $10 \mathrm{ppm}$ par micron d'oxyde. Les oxyda- 
tions relevées ne conduisent habituellement pas à des teneurs en hydrogène qui pourraient entraîner une dégradation majeure du gainage ou des composants de structure.

Toutefois, des oxydations importantes peuvent être très exceptionnellement constatées lorsque la gaine d'un crayon n'est plus étanche (défaut de fabrication, corps migrants, frottement dans une grille...).

Ainsi, actuellement, on ne constate pas de problèmes de corrosion des matériaux de gainage ; cependant, pour des raisons évidentes, les exploitants souhaitent prolonger l'irradiation du combustible jusqu'à des combustions massiques moyennes de l'ordre de $47 \mathrm{GWj} / \mathrm{tU}$ voire $52 \mathrm{GWj} / \mathrm{tU}$. Pour ce faire, la preuve du maintien de l'intégrité de la première barrière doit être apportée. Des assemblages caractérisés font, pour cette raison, l'objet d'une surveillance toute particulière et leur irradiation est prolongée jusqu'à 5 cycles.

Les examens sur site ont montré qu'après de telles irradiations les gaines de crayons de combustible s'oxydent fortement, les couches d'oxyde sont moins adhérentes et un écaillage ou une desquamation est souvent observée (Fig. 1).

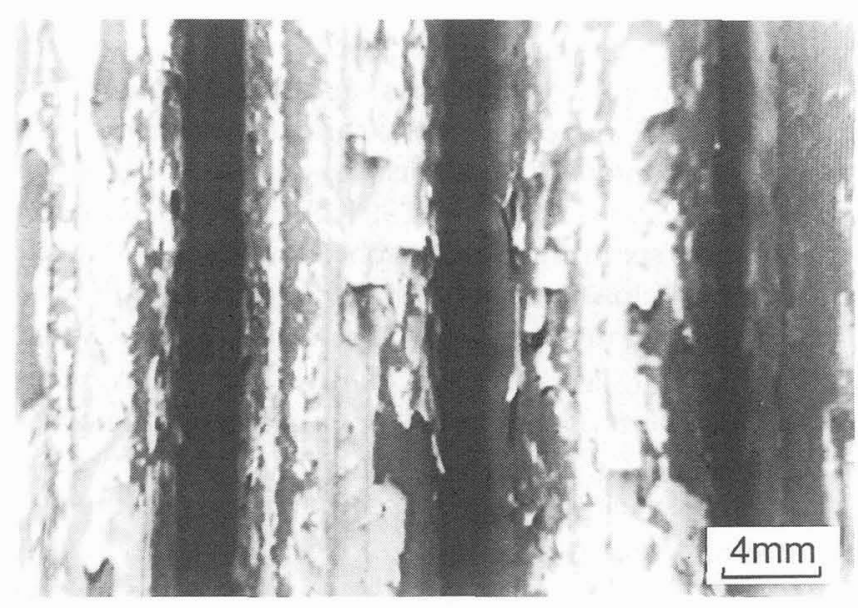

Fig. 1. - Examen visuel sur site des crayons périphériques d'un assemblage irradié 5 cycles.

Les couches d'oxyde mesurées en cellule chaude au CEA dans un cadre de recherche et développement sont de l'ordre de $100 \mu \mathrm{m}$ [3]. De plus, l'épaisseur de gaine restante très irradiée est fortement hydrurée. Dans le but de démontrer qu'en dépit de cette oxydation des caractéristiques mécaniques satisfaisantes pour garantir l'objectif de sûreté sont conservées, des examens doivent être prochainement menés. Ces examens devront être complétés par l'étude du comportement du gainage en condition accidentelle d'APRP (Accident par Perte de Réfrigérant Primaire).

Les résultats de ces études conditionnent la généralisation de l'utilisation du combustible nucléaire de conception actuelle jusqu'à de forts épuisements spécifiques. Parallèlement, depuis quelques années, les fournisseurs étudient et expérimentent des combustibles de nouvelle conception, notamment sur le plan des matériaux et de leur tenue à la corrosion, qui permettront leur utilisation sans risque pendant au moins 5 cycles. 
2.2 LE CCEUR ET LES INTERNES. - Il est admis que les matériaux, quels qu'ils soient, subissent sous une irradiation neutronique importante des dommages divers. Ainsi, les aciers faiblement alliés constitutifs de la cuve, particulièrement pour les parties situées au droit du cœur, peuvent à terme perdre une partie de leurs bonnes caractéristiques mécaniques telle leur température de transition fragile/ductile qui peut augmenter notablement. Ceci est bien connu et chaque centrale est suivie grâce à des éprouvettes installées dans le cœur et prélevées à intervalles définis pour connaître le vieillissement de la cuve.

Par contre, bien que ce phénomène ait déjà été noté antérieurement, il semble que des dommages spécifiques aux aciers inoxydables austénitiques sous irradiation neutronique soient constatés plus souvent ces derniers temps. Deux exemples récents viennent d'être rencontrés en France.

Il s'agit de la rupture intergranulaire de ces matériaux dans des milieux a priori non corrosifs et sous des contraintes de tension à priori acceptables.

L'un de ces exemples, mis en évidence début 1989, concerne la fissuration des vis de cloisonnement des internes inférieurs. Le cloisonnement est un ensemble assemblé par vis qui permet de réaliser la transition entre le contour polygonal du cœur et l'enveloppe du cœur cylindrique.

A la suite d'un incident sur un assemblage combustible, mis sur le compte d'une détérioration du cloisonnement qui ne jouait plus son rôle protecteur vis-à-vis de la différence de pression existant de part et d'autre, une vaste campagne d'investigation a été lancée. En particulier, une méthode de contrôle par ultrasons a été mise au point pour vérifier l'état des vis. Les contrôles effectués entre 1989 et 1991 ont permis de dénombrer la fissuration d'un peu plus de 70 vis sur les six premières tranches du palier $900 \mathrm{MW}$ (960 vis par cloisonnement) ; les suivantes, dont le cloisonnement est de conception différente, ne présentent pas de vis fissurées. A la suite de ces contrôles, 5 vis ont été extraites et expertisées au laboratoire.

Les examens ont montré que la fissuration était intergranulaire (Fig. 2) et que les caractéristiques mécaniques n'avaient pas été fortement affectées par l'irradiation (augmentation de la limite d'élasticité de $140 \mathrm{MPa}$, allongement à rupture encore égal à 25\%). Ces résultats ne sont pas en désaccord avec les fluences subies par les vis, estimées de l'ordre de 0,9 à $2,6 \times 10^{22} \mathrm{n} / \mathrm{cm}^{2}$, plutôt inférieures à la fluence nécessaire pour diminuer notablement la ductilité du matériau [4]. Ainsi, les dommages d'irradiation seuls ne semblent pas suffisants pour expliquer la fissuration intergranulaire des vis même si des examens récents en microscopie électronique en transmission montrent certains dommages comme la disparition de la structure initiale de dislocation et l'apparition de très nombreuses petites boucles de dislocation $(20 \mathrm{~nm})$.

Pourtant, l'analyse des corrélations entre fluence, température, efforts et les vis fissurées montre un accord satisfaisant puisque l'on peut définir un domaine de sensibilité à la fissuration des vis en fonction de la contrainte et de l'irradiation.

Dans l'état actuel des connaissances, on peut également penser à un phénomène de corrosion fissurante assistée par l'irradiation [5] (IASCC - Irradiated Assisted Stress Corrosion Cracking) dont le mode d'action peut être très variable ; soit en jouant sur le milieu chimique (radiolyse de l'eau, concentration d'espèces nocives par surchauffe...) ; soit sur le matériau (diffusion des lacunes et des atomes, ségrégation d'impuretés aux joints de grain, déchromisation).

Le second exemple concerne la fissuration des gaines des crayons de grappe de commande qui sont en acier AISI 304. Ces crayons qui contiennent un absorbant neutronique (Alliage Ag-In-Cd, $\mathrm{B}_{4} \mathrm{C}$ ) servent à régler la puissance du cœur et permettent, en cas de problème, l'arrêt immédiat par leur chute et de stopper ainsi la réaction de fission. 


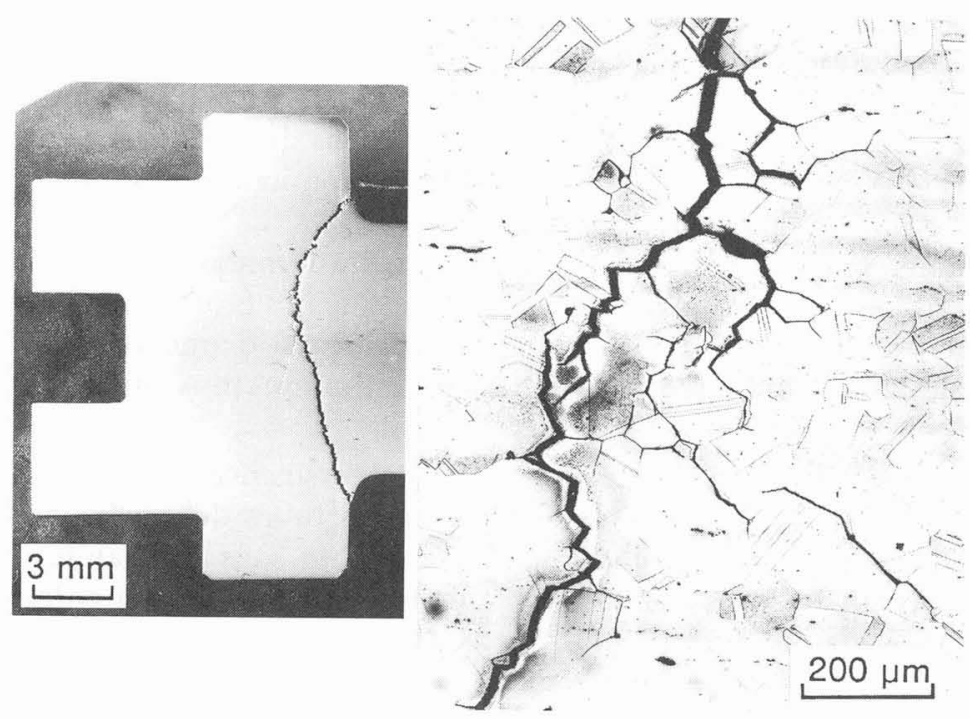

Fig. 2. - Examen micrographique au niveau du congé tête-fût d'une vis de cloisonnement de cœur.

Certaines de ces gaines se fissurent au niveau de l'ogive inférieure après avoir subi un léger gonflement. Celui-ci est dû au tassement de l'absorbant en extrémité inférieure et à la diminution de densité de ce dernier sous l'effet de l'irradiation par les neutrons (transmutation de l'indium en étain).

Des expertises réalisées récemment ont montré que la fissuration était intergranulaire (Fig. 3). Comme pour les vis de cloisonnement, a priori, les dommages d'irradiation étaient considérés comme trop faibles pour justifier cette rupture intergranulaire. Cependant, des essais de fluage à $450^{\circ} \mathrm{C}$ ont reproduit cette fissuration sur des matériaux de gainage irradiés et non sur du matériau non irradié.

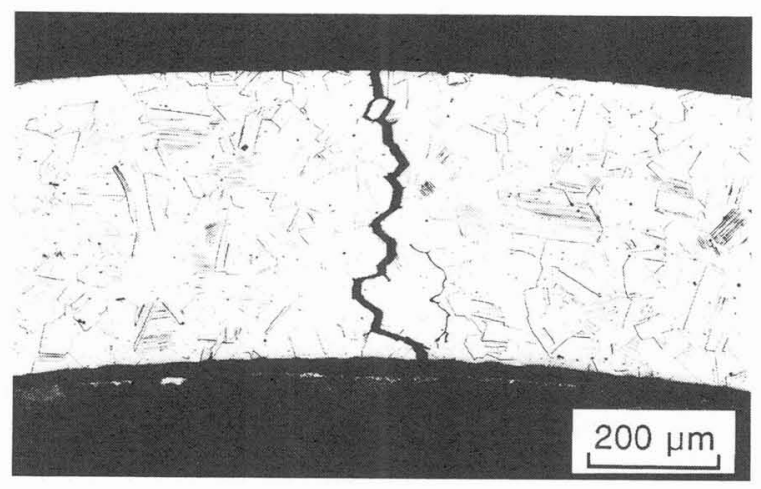

Fig. 3. - Fissuration d'une grappe de commande. 
Ainsi, il semblerait que pour les gaines, la fissuration soit due essentiellement aux dommages d'irradiation qui fragilisent le matériau et qu'il suffit d'une légère déformation pour le rompre intergranulairement ; dans le cas des vis de cloisonnement, les dommages d'irradiation seraient insuffisants et il faudrait une concomitance avec un autre phénomène comme l'IASCC pour expliquer les ruptures.

Ces comportements sous irradiation des matériaux austénitiques - fragilisation, corrosion assistée par l'irradiation - font partie de nos soucis majeurs et une meilleure connaissance de ces phénomènes grâce aux études engagées permettra d'améliorer la conception des nouvelles centrales (irradiation maximale permise, contraintes plus faibles, confinement, ...).

2.3 LE CIRCUIT PRIMAIRE. - Un des problèmes majeurs rencontrés par les exploitants de réacteurs à eau pressurisée concerne la fissuration des tubes de générateur de vapeur en alliage 600 . La sensibilité de ce matériau à la corrosion sous contrainte dans l'eau pure à des températures supérieures à $300^{\circ} \mathrm{C}$ et sous forte contrainte, est connue depuis 1959 [6]. Les premières manifestations dans les appareils en fonctionnement ont eu lieu en 1970 et se sont depuis répandues universellement. Ceci contraint les exploitants à de très nombreux contrôles, aux bouchages d'un grand nombre de tubes et même au remplacement des générateurs de vapeur. Des actions préventives et des solutions de remplacement ont été, maintenant, mises au point pour régler ce problème.

Par contre, depuis quelques années, d'autres matériels du circuit primaire, constitués de pièces en alliage 600 plus massives que les tubes de générateur de vapeur, commencent également à se fissurer.

En France, la première alerte s'est produite en 1989 sur les piquages d'instrumentation des pressuriseurs des centrales 1300 MW. Lors du renouvellement d'épreuve hydraulique du circuit primaire principal, des fuites (suintement de quelques gouttes) sont apparues sur deux centrales à la pression d'épreuve (207 bar). Les expertises effectuées sur des piquages défectueux ont mis en évidence un réseau de fissures de plusieurs mm de longueur affectant la peau interne du tube en alliage 600 à proximité de la soudure du piquage sur le pressuriseur.

Cette fissuration provient d'un phénomène de corrosion sous contrainte de l'alliage 600 dans le fluide primaire identique à celui constaté sur les générateurs de vapeur, les contraintes de tension responsables étant les contraintes résiduelles subsistant après le dudgeonnage et le soudage du piquage dans le pressuriseur.

Compte tenu du caractère générique et des risques, certes faibles, mais réels d'éjection d'un piquage, une stratégie a été mise en œuvre de manière à aboutir en deux ans au remplacement de l'ensemble des piquages en alliage 600 par des piquages en acier austénitique $18 \mathrm{Cr}-10 \mathrm{Ni}$.

Parallèlement à ces réparations, une réflexion a été engagée, avec le constructeur, pour établir un bilan des alliages à base de nickel utilisés dans le circuit primaire principal. Ensuite, une analyse des risques associés à la corrosion sous contrainte de l'alliage 600 dans les zones les plus critiques du circuit primaire principal a été entreprise en intégrant les multiples paramètres de la construction et des conditions de service qui peuvent intervenir - comme le matériau - les contraintes résiduelles et de service et la température.

Pour ceci, il a fallu définir une méthode qui permette de porter un jugement sur les risques d'amorçage de fissures par corrosion sous contrainte dans les composants en alliage de nickel du circuit primaire principal. Cette méthode a été établie en utilisant le retour d'expérience et les résultats de laboratoire.

Des indices de sensibilité ont été attribués au matériau [7] (influence de la structure métallurgique), à la température (loi du type Arrhenius avec une énergie d'activation de 
$44 \mathrm{kcal} / \mathrm{mole}$ ) et à la sollicitation mécanique (temps d'amorçage de la fissuration proportionnel à la contrainte principale à la puissance -4 , en tenant compte pour les plus faibles contraintes de l'écrouissage du matériau).

Cette première analyse a permis de classer sur une échelle de risque de fissuration les différentes pièces du circuit primaire principal constituées d'alliage de nickel. Elle a permis également, compte tenu d'incertitudes sur certaines données comme le niveau de contrainte résiduelle, de lancer des études pour affiner ce classement. Enfin, des priorités ont pu être établies vis-à-vis de la mise au point de méthodes de contrôles non destructifs nécessaires pour vérifier le comportement des pièces susceptibles de fissurer durant la vie de la centrale.

Cette étude à peine terminée, un deuxième cas de fissuration de pièces en alliage $600 \mathrm{du}$ circuit primaire principal s'est produit en fin d'année 1991.

A l'occasion d'une épreuve hydraulique, dans le cadre d'une visite décennale, un léger écoulement d'eau a été constaté à la pression de 207 bar sur le bord du couvercle de la cuve de Bugey 3.

Les examens visuels et télévisuels ont permis de confirmer que la fuite provenait d'un adaptateur. Cet adaptateur qui est une gaine en alliage 600 , fretté et soudé sur le couvercle permet le passage de la tige de commande d'une grappe de contrôle.

Après dépose du couvercle de cuve et démontage du mécanisme de grappe, des examens par ressuage, courants de Foucault, ultrasons, ont mis en évidence des fissures longitudinales en partie basse de l'adaptateur au voisinage de la zone de soudure du couvercle. Des expertises métallurgiques sont prévues pour confirmation, mais il est indubitable qu'il s'agit, là encore, d'une corrosion sous contrainte de l'alliage 600 dans le milieu primaire.

Bien entendu, cette pièce avait été prise en compte dans l'étude des zones à risque du circuit primaire principal. Et, s'il est vrai qu'elle n'avait pas été classée comme la zone la plus à risque, la probabilité de fissuration de cette zone ne pouvait pas être négligée.

D'autre part, il était admis que des essais sur maquettes devaient être entrepris pour améliorer nos connaissances sur le niveau des contraintes de tension. Cette dernière remarque était justifiée puisqu'en effet, au cours des examens qui ont suivi les premières constatations sur Bugey 3, il s'est avéré que le temps plus court d'apparition de la fissuration que celui attendu, découlait d'un niveau de contrainte au niveau de la soudure plus élevé que celui pris en compte pour établir le classement ; en particulier, pour les adaptateurs situés en périphérie dont la soudure est très dissymétrique.

Une des retombées de ces affaires est que nous avons maintenant un outil, certes qui doit être encore amélioré, capable de déceler les zones qui peuvent présenter des risques durant les 40 années de vie prévues de la centrale. Ceci va entraîner bien évidement une agmentation notable des contrôles et des opérations de maintenance avec la mise au point, préventivement, de méthodes de contrôles adaptées et de procédés de réparation adéquats. Une autre retombée évidente est bien entendu, l'utilisation d'alliages différents comme les aciers austénitiques et l'alliage 690 pour les pièces soumises à des contraintes de tension dans le milieu primaire, dans le cas des nouvelles tranches en cours de construction.

2.4 LE CIRCuIt SECONDAIRE . - Dès le début des années 60, on s'est inquiété des risques de corrosion sous contrainte des alliages austénitiques utilisés pour les tubes de générateur de vapeur. Pour les alliages de nickel, ce risque fut circonscrit à la corrosion caustique et des études intensives furent entreprises.

Celles-ci permirent de connaître assez bien le rôle des différents paramètres comme la contrainte, la température ou la concentration en soude (Fig. 4) [8].

Par ailleurs, les connaissances sur la chimie et la thermohydraulique dans les crevasses se sont également fortement améliorées. 


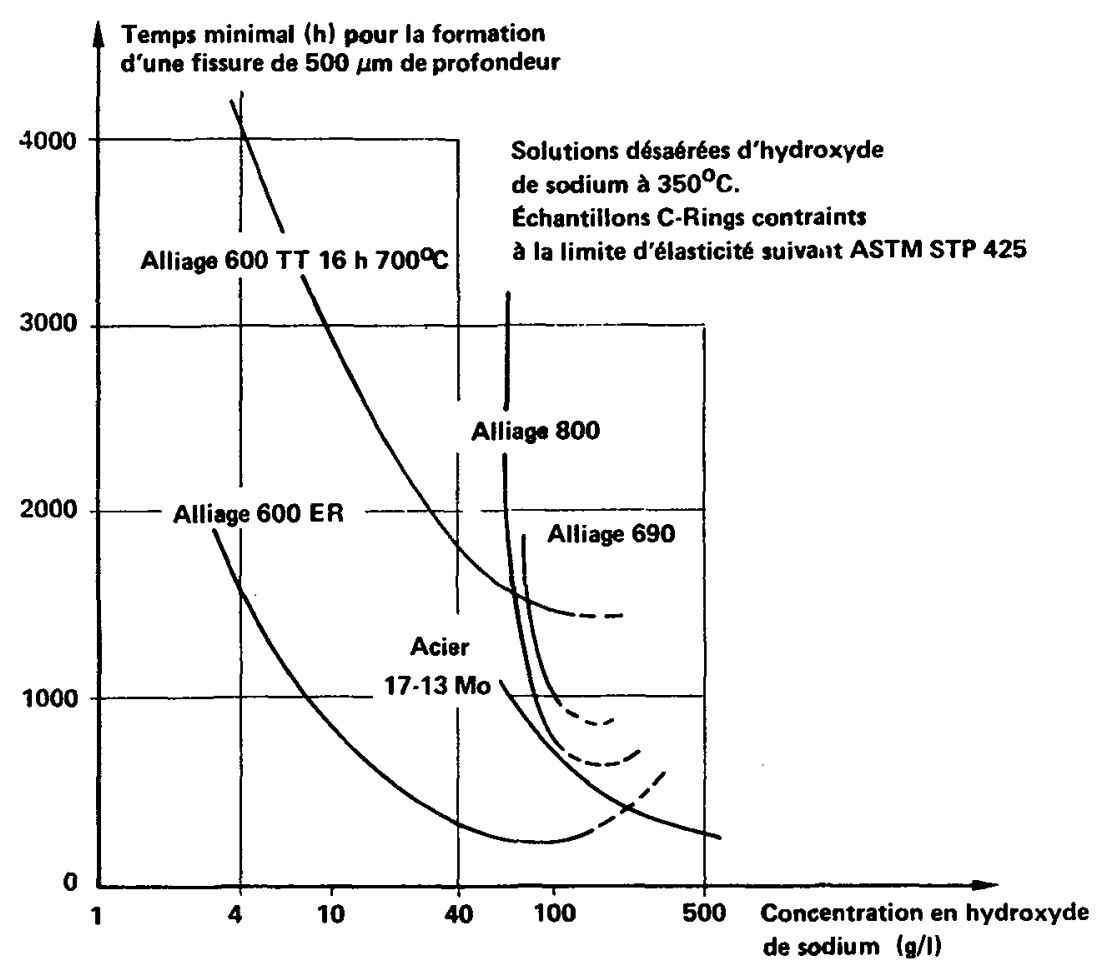

Fig. 4. - Comportement des alliages austénitiques dans la soude à $350^{\circ} \mathrm{C}$.

L'ensemble de ces études a permis de prendre des mesures correctives qui pouvaient nous laisser espérer que ce risque était pratiquement éliminé si l'exploitant respectait les spécifications.

Malheureusement, malgré les spécifications chimiques actuelles, à la limite de ce qui est possible d'imposer, des fissurations du côté secondaire continuent de se produire dont certaines sont difficiles à expliquer par la présence de soude caustique seule le long des tubes.

Ainsi, depuis 1988 en France, on constate la présence significative de plomb sur des tubes extraits présentant des fissures côté secondaire. Dans certains cas, la présence de plomb a même été décelée dans les fissures. Les teneurs en plomb peuvent atteindre plusieurs pourcents dans les dépôts. D'autre part, le faciès de la fissuration est souvent particulier puisqu'il est à la fois inter et transgranulaire (Fig. 5).

Ces observations ont donc conduit à lancer une étude dont les actions principales sont :

- connaître l'agressivité des composés du plomb vis-à-vis des matériaux ;

- connaître la nature des composés du plomb pouvant être présents dans le circuit eauvapeur ;

- apprécier les sources potentielles de plomb pouvant expliquer les fortes teneurs parfois observées dans les dépôts ;

- mettre au point des remèdes.

En ce qui concerne l'agressivité du plomb, de nombreuses études ont été lancées et il est indéniable que certains composés comme $\mathrm{PbO}$ sont, particulièrement en milieu caustique, un facteur d'accélération du risque de corrosion sous contrainte des alliages austénitiques [9]. 


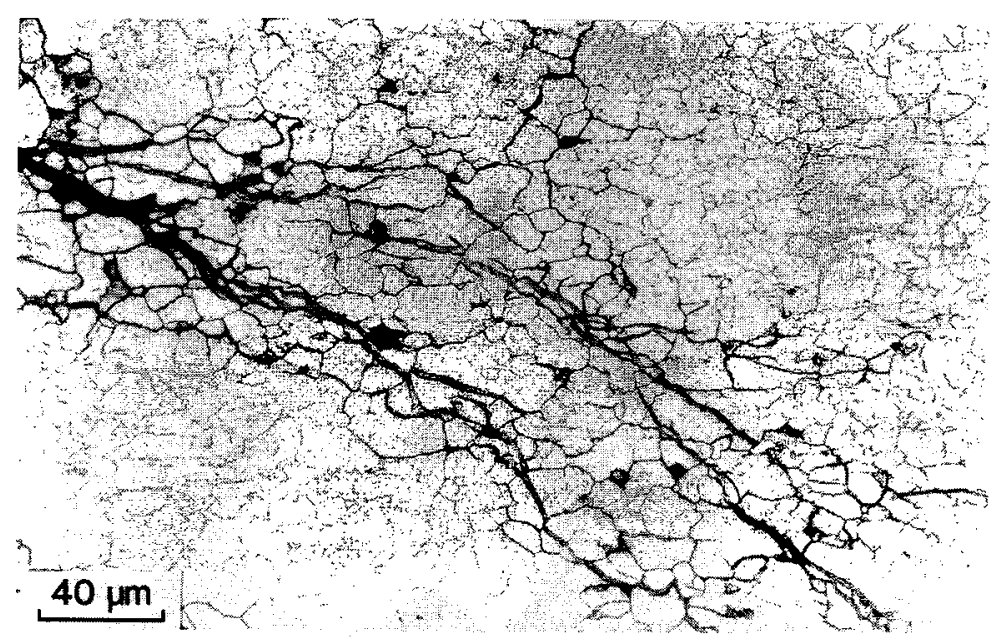

Fig. 5. - Tube de générateur de vapeur. Fissuration mixte (inter/trans) granulaire.

Le mécanisme $[10,11]$ invoqué suggère que le plomb et l'oxyde de plomb réagissent avec les couches d'oxydes des alliages de nickel pour réduire l'oxyde de nickel et former des produits de type chromate de plomb.

$$
\begin{array}{cl}
2 \mathrm{~Pb}+5 \mathrm{NiO}+\mathrm{Cr}_{2} \mathrm{O}_{3} & \longrightarrow 2 \mathrm{PbCrO}+5 \mathrm{Ni} \\
2 \mathrm{PbO}+2 \mathrm{OH}^{-} & \longrightarrow 2 \mathrm{HPOO}_{2}^{-} \\
2 \mathrm{HPbO}_{2}^{-}+3 \mathrm{NiO}+\mathrm{Cr}_{2} \mathrm{O}_{3} & \longrightarrow 2 \mathrm{PbCrO}^{2}+3 \mathrm{Ni}+20 \mathrm{H}^{-}
\end{array}
$$

Cette transformation de la couche d'oxyde de nickel diminuerait le temps d'incubation de la fissuration. Pour ce qui concerne la présence de composés du plomb dans les générateurs de vapeur, il a été montré que ce sont les espèces $\mathrm{Pb}$ et $\mathrm{Pb}(\mathrm{OH})_{2}$ qui sont les espèces les plus stables à $300^{\circ} \mathrm{C}$. D'autre part, des mesures en centrale ont montré que la teneur en plomb de l'eau d'alimentation ne dépassait pas $40 \mathrm{ppt}(\mu \mathrm{g} / \mathrm{t})$ ce qui représente au maximum $0,1 \mathrm{~g}$ de plomb par heure apporté dans le générateur de vapeur. D'autres sources sont donc nécessaires et les inventaires qui ont été effectués semblent indiquer que les pollutions viendraient très probablement de la turbine (plombage, huiles, protections temporaires).

A ce sujet, il a été montré qu'une pollution par le plomb, au niveau du condenseur ou de la turbine, pouvait conduire à ce que les dépôts sur les tubes, au niveau des interstices tubeplaque, aient des teneurs en plomb beaucoup plus importantes que ceux situés sur les parties libres du tube. Ceci a été corroboré par des analyses sur des tubes extraits qui montrent clairement que la teneur en plomb est plus forte dans les dépôts du tube situés dans les interstices (Fig. 6).

Pour résoudre ce problème, constaté aujourd'hui essentiellement sur les tranches les plus anciennes ( $>10$ ans), des mesures vont être prises pour limiter les risques de pollution et, pour les centrales déjà polluées, mettre au point un procédé de nettoyage chimique qui dissolve les composés du plomb déposés sur les tubes. 


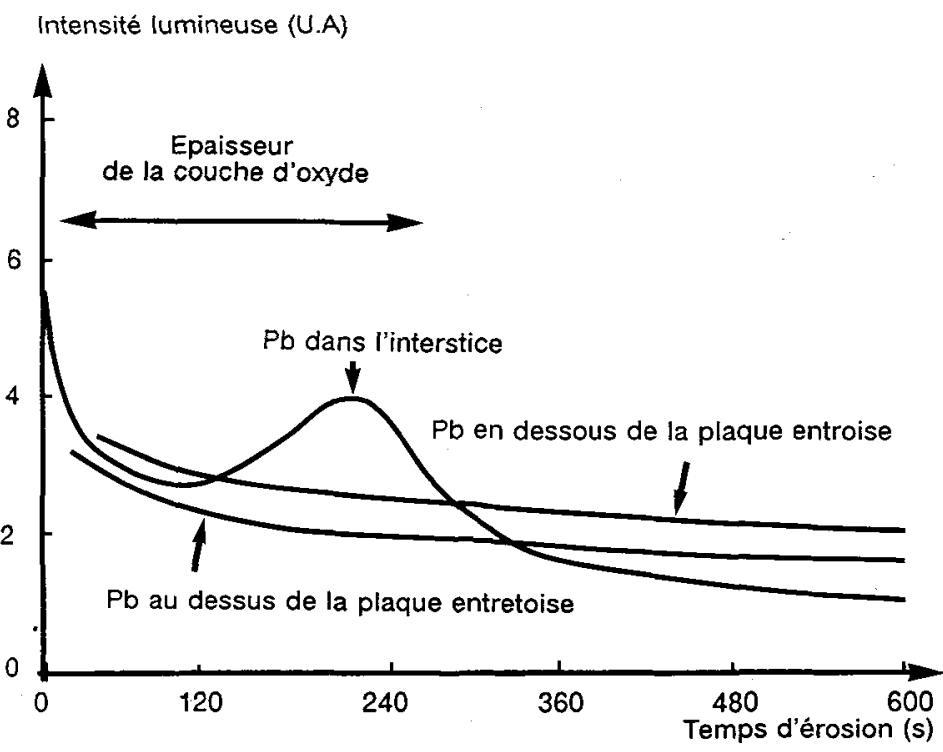

Fig. 6. - Analyse par spectrométrie à décharge luminescente sur un tube de générateur de vapeur.

\section{Conclusion.}

Les centrales à eau pressurisée après avoir eu des maladies de jeunesse souvent causées par des phénomènes de corrosion ont bénéficié très rapidement d'actions specifiques pour remédier aux dégradations constatées. Ces actions ont été centrées sur la qualité de l'eau et la propreté des chantiers pendant la construction ou les opérations de maintenance. Elles ont également nécessité la mise au point de méthodes de contrôles et de réparations et le choix de matériaux plus adaptés aux conditions sévères de fonctionnement.

Cependant, aujourd'hui, du fait du vieillissement des centrales, d'autres phénomènes de corrosion se font jour, soit parce que les matériels concernés sont dans un environnement moins sévère que celui qui régnait autour des matériels qui ont eu des problèmes dès les premières années, soit parce qu'il fallait attendre une dizaine d'années pour créer les conditions nécessaires à l'apparition de la corrosion.

Ces nouveaux problèmes, conduisent, comme pour les maladies de jeunesse, à des études pour comprendre les mécanismes et mettre au point des solutions pour éviter la continuation ou la reproduction des dégradations. Ils obligent également à augmenter les opérations de maintenance et à mettre en œuvre des méthodes de contrôles non destructifs de plus en plus sophistiquées et de plus en plus sensibles.

Ces constatations montrent la difficulté inhérente à des installations comme les centrales nucléaires puisque des choix de matériaux ou de conception effectués il y a 20 ans commencent seulement aujourd'hui à montrer qu'ils n'étaient pas parfaitement adaptés aux conditions de fonctionnement. Pour combattre cette difficulté, une exploitation intelligente et complète du retour d'expérience, associée aux études de laboratoire, apparait être la meilleure solution. 


\section{Bibliographie}

[1] GRAS J.M., Etude bibliographique de la corrosion généralisée des alliages zircaloy dans les conditions intéressant le fonctionnement des réacteurs à eau légère, Procèsverbal EDF/EMA HT-45 n564 (15 Mars 1988).

[2] GOURMELON, Influence de la composition chimique et du procédé de fabrication sur la corrosion aqueuse en réacteur des crayons de combustible. Comportement des fournitures livrées à EDF Note D. 342 DRTN 88.177 GRN/FL (18 Mai 1988).

[3] GuEdenEy P. et Coll., Caractérisation du comportement du combustible Fragéma à fort taux de combustion, International Topical Meeting on LWR Fuel Performance (Avignon, April 21-24, 1991).

[4] VAN DUYSEN J.C., Etude bibliographique des effets de l'irradiation sur les caractéristiques mécaniques et la microstructure de l'acier Z6 CND 17-12 écroui HT41/NEQ 1172-A (EDF, Etudes et Recherches).

[5] ANDRESEN P.L., FORD F.P., MURPHY S.M., PERKS J.M., State of knowledge of radiation effects on environmental cracking in light water reactor core materials, Proceedings of the Fourth International Conference on Environmental Degradation of Materials in Nuclear Power Systems Water Reactors (Jekyll Island, GA, August 1989) (NACE, 1990).

[6] Coriou H., Grall L., Mahieu C., Pelras M., Corrosion 22 (1966) 280.

[7] BOUdOT R., VIDAL P., GElPI A., GimOND C., Zones en inconel du circuit primaire principal des REP. Méthodes d'évaluation de la sensibilité à la corrosion sous contrainte des alliages à base de nickel, rapport EDF-DER - HT-40/NEQ 1273-B.

[8] BERGE Ph., DONATI J.R., Nucl. Technol. 55 (October 1981) 88.

[9] Rapport EPRI NP 7367-M (July 1991).

[10] MIGLIN B.P., SARVER J.M., Investigation of lead as cause of IGA at support plate intersections (Congrès EPRI, 1990).

[11] SAKAI T., AOKI K., KISHI Y., Effect of water chemistry on lead induced stress corrosion cracking of alloy 600 (Colloque International de Fontevraud, SFEN, Septembre 1990). 